\title{
Frontal brain lobe impairment in obstructive sleep apnoea: a proton MR spectroscopy study
}

\author{
M. Alchanatis*, N. Deligiorgis*, N. Zias*, A. Amfilochiou\#, E. Gotsis", A. Karakatsani*, A. Papadimitriou+
}

Frontal brain lobe impairment in obstructive sleep apnoea: a proton MR spectroscopy study. M. Alchanatis, N. Deligiorgis, N. Zias, A. Amfilochiou, E. Gotsis, A. Karakatsani, A. Papadimitriou. (C) ERS Journals Ltd 2004.

ABSTRACT: Patients with obstructive sleep apnoea syndrome frequently have cognitive deficits, especially related to executive functions, which cannot be fully explained by daytime sleepiness and are partial irreversible after nasal continuous positive airway pressure treatment. The causal mechanism of these cognitive deficits is not yet known, but it has been proposed that they are associated with chemical and structural brain cell injury. The aim of this study was to investigate brain metabolism in patients with sleep apnoea syndrome.

Twenty-two patients with severe sleep apnoea and 10 healthy volunteers of comparable age were studied using single voxel proton magnetic resonance spectroscopy. Magnetic resonance spectra were obtained from prefrontal cortex, parietooccipital and frontal periventricular white matter.

$\mathrm{N}$-acetylaspartate-to-creatine and choline-to-creatine ratios were significantly lower in the frontal white matter of obstructive sleep apnoea patients when compared to controls. Absolute concentrations of $\mathrm{N}$-acetylaspartate and choline were also significantly reduced in the frontal white matter of patients with sleep apnoea.

Frontal lobe white matter lesions are known to be associated with cognitive executive dysfunction. The findings of this study may offer an explanation for the sometimes irreversible cognitive deficits associated with sleep apnoea.

Eur Respir J 2004; 24: 980-986.
*Sleep Laboratory, Pulmonary Dept, University of Athens Medical School, "SOTIRIA" Hospital for Diseases of the Chest, "Sleep Laboratory, "Sismanoglio" Hospital, and Encephalos Institute, Athens, and ${ }^{+}$Neurology Dept, University of Thessalia Medical School, Larissa, Greece.

Correspondence: M. Alchanatis

10, Bakopoulou Str.

15451 Athens

Greece

Fax: 302107770423

E-mail: annakara@otenet.gr

Keywords: Cerebral metabolism proton magnetic resonance spectroscopy sleep apnoea syndrome

Received: November 152003

Accepted after revision: August 122004
Obstructive sleep apnoea (OSA) syndrome (OSAS) is a common disorder occurring in up to $4 \%$ of the general adult population [1]. Cognitive impairment has been repeatedly reported in OSA patients. The areas most frequently affected are general intellectual functioning, attention, memory and, in particular, executive functions, such as problem solving, planning of goal-oriented behaviour and mental flexibility $[1,2]$. To date, the pathophysiology of the cognitive deficits reported in OSA patients has not been determined. Some researchers argue that excessive daytime somnolence is the leading cause of the cognitive deficits, while others propose that nocturnal hypoxaemia is the main contributing factor $[1,2]$. Moreover, several studies have shown that executive dysfunction in OSA patients may persist even after nasal continuous positive airway pressure (nCPAP) treatment [2-4]. Cognitive executive functions are associated with specific prefrontal-subcortical brain circuits [5], thus it has been proposed that OSAS may promote irreversible anoxic brain damage affecting the prefrontal cortex $[4,6]$.

Proton magnetic resonance spectroscopy $\left({ }^{1} \mathrm{H}\right.$ MRS) provides a noninvasive in vivo assessment of brain metabolism. ${ }^{1} \mathrm{H}$ MRS has been applied to the study of many brain disorders, including Alzheimer's disease, brain tumours, epilepsy, multiple sclerosis, leukodystrophies and mitochondrial disorders [7, 8].

The aim of this study was to detect possible metabolic abnormalities in the brain of patients with severe OSAS using single voxel ${ }^{1} \mathrm{H}$ MRS. Both the prefrontal cortex and the frontal periventricular white matter were examined, as these regions are associated with executive cognitive dysfunction [5], which is frequently reported in OSA patients. The parietooccipital periventricular white matter was also examined, as spectroscopic abnormalities have been previously reported in OSA patients $[9,10]$.

\section{Materials and methods}

\section{Study subjects}

Twenty-two consecutive patients with severe OSAS who fulfilled the following inclusion criteria were enrolled in this study: apnoea/hypopnoea index (AHI) $>30$, age $<65 \mathrm{yrs}$, no history of stroke, and absence of neurological disease or history of head injury. Patients with claustrophobia or metallic implants were excluded. All patients were diagnosed in the Sleep Laboratory of the Pulmonary Dept of the Medical School (Athens University), in "Sotiria" Hospital for Chest Diseases, or in the Sleep Laboratory of "Sismanoglio" Hospital (both Athens, Greece), between June and December 2002. Ten healthy male volunteers of comparable age were used as the control group. The Ethics Committees of both hospitals approved the study, and all patients and control subjects gave written informed consent. 


\section{Study design}

All subjects underwent a full-night polysomnography (Embla, Flaga ht; Medical Devices, Reykjavik, Iceland), with recordings of the following: electroencephalogram; electrooculogram; chin electromyogram; electrocardiogram; oral and nasal flow; abdominal and thoracic movements; oxyhaemoglobin saturation; and snoring [11]. Excessive daytime sleepiness was assessed by using the Epworth Sleepiness Scale (ESS). Within 2 weeks of polysomnography and before the initiation of nCPAP treatment, all eligible patients, as well as control subjects, underwent single voxel brain ${ }^{1} \mathrm{H}$-MRS.

\section{Methods}

Spectroscopy was performed at 1.5 Tesla using PROBE (Signa Hispeed; General Electric, Milwaukee, WI, USA). Prior to ${ }^{1} \mathrm{H}$ MRS, axial T1-weighted and FLAIR (fluidattenuated inversion recovery) images were obtained, in order to exclude neurological disease and to localise the voxels. Two OSA patients had punctuated white matter hyperintensities. Voxels were carefully placed outside these lesions, in normalappearing brain tissue. No magnetic resonance imaging abnormalities were found in the control group. Proton spectra were obtained from: 1) right parieto-occipital white matter, next to the occipital horn of the lateral ventricle; 2) left frontal white matter, next to the frontal horn of the lateral ventricle; and c) left prefrontal cortex (figs $1 \mathrm{a}, 2 \mathrm{a}$ and $3 \mathrm{a}$ ).

A single voxel spin-echo PRESS (point-resolved spectroscopy) sequence was used with echo time $(t \mathrm{E}) 35 \mathrm{~ms}$, repetition time $(t \mathrm{R}) 1,500 \mathrm{~ms}$ and 96 signal acquisitions, resulting in an acquisition time of $3 \mathrm{~min}$ per pixel. The choice of single-voxel MRS instead of chemical-shift imaging (CSI) was made for two reasons: first, in order to assure accurate placement of the volume of interest and to avoid signal contamination with unwanted tissue; and, secondly, in order to assure higher accuracy in quantitation, knowing that significant quantitation differences exist across all voxels of a CSI slab. The choice of $t \mathrm{R} / t \mathrm{E}$ was made basically for signal-tonoise ratio optimisation for the given spectral acquisition time. Voxel volumes were $6.7 \pm 1.8 \mathrm{~cm}^{3}$ (mean $\pm \mathrm{SD}$ ) for the frontal white matter, $8.2 \pm 2.4 \mathrm{~cm}^{3}$ for the posterior white matter and $5 \pm 0.8 \mathrm{~cm}^{3}$ for the frontal grey matter. This variability in volumes was necessary in order to ensure that, in spite of individual anatomical differences, only white or only gray matter were included in each voxel. Voxels were placed in the same regions for all subjects, by the same experienced investigator (E. Gotsis), to decrease intra-subject variation. Peaks corresponding to $N$-acetylaspartate (NAA), creatine $(\mathrm{Cr})$, choline (Cho) and myo-inositol ( $\mathrm{mI}$ ) were measured, and the NAA/Cho, NAA/Cr, Cho/Cr and $\mathrm{mI} / \mathrm{Cr}$ ratios were calculated [12]. The absolute concentrations of NAA, Cho, $\mathrm{Cr}$ and $\mathrm{mI}$ were also calculated, with direct comparison of patient spectra to the spectrum of an external phantom of known metabolite concentrations (GE phantom of known concentrations), correcting for filling factor differences of the coil for each patient, as well as for the phantom (actually using the head coil transmitter gain in each case). Concentrations were calculated in mmol per $\mathrm{kg}$ wet tissue. The use of metabolite ratios in MRS studies reduces systematic errors, because they would affect all metabolites for a particular voxel in the same proportion. However, this method cannot distinguish between numerator and denominator changes. Conversely, the calculation of absolute metabolite concentrations, although sensitive to technical errors, can be very helpful for the interpretation of ratio changes.

\section{Analysis}

All values are expressed as mean \pm SD. The Mann-Whitney U-test was used to compare age, metabolite ratios and concentrations between the two groups. This nonparametric test was chosen because of the small sample size and the lack of previous data showing a normal distribution for the examined ratios and concentrations in OSA patients. Correlations between metabolite ratios, metabolite concentrations and specific respiratory parameters, ESS and age, were determined using Spearman's correlation. A p-value $<0.05$ was considered statistically significant. A general linear model test (multivariate ANOVA) was used in order to examine the effect of interactions of age, ESS, AHI, minimal nocturnal oxyhaemoglobin saturation $\left(\mathrm{Sa}_{\mathrm{a}} \mathrm{O}_{2}, \mathrm{~min}\right)$, mean nocturnal oxyhaemoglobin saturation $\left(\mathrm{Sa}_{\mathrm{a}}, \mathrm{O}_{2}\right.$, mean $)$ and absolute time of oxyhaemoglobin saturation $<90 \%$ during sleep $(t<90 \%)$ on metabolite ratios and concentrations.

\section{Results}

The clinical characteristics of OSA patients and control subjects, as well as statistical comparisons between them, are presented in table 1. Fourteen out of the 22 patients did not have cardiac disease or cardiovascular risk factors, including hypertension, hyperlipidaemia and diabetes mellitus, according to their medical history. Out of the eight remaining patients, one had ischaemic heart disease, one had atrial fibrillation, six had hypertension, two had hyperlipidaemia and two had diabetes mellitus, all under medical treatment.

In comparison to the control group, OSA patients showed a significant reduction in the NAA/Cr and $\mathrm{Cho} / \mathrm{Cr}$ ratios in frontal white matter $(\mathrm{p}=0.012$ and $\mathrm{p}=0.008$, respectively). Table 2 summarises the results of the comparison of the metabolite ratios between OSA patients and controls in the three examined areas. Figures $1 b, 2 b, 3 b$ and $1 c, 2 c, 3 c$ display representative spectra of one OSA patient and one control subject, respectively. In figure 4, scatter plot diagrams of $\mathrm{NAA} / \mathrm{Cr}$ and $\mathrm{Cho} / \mathrm{Cr}$ ratios in frontal white matter are shown. OSA patients had significantly decreased NAA and Cho absolute concentrations in frontal white matter compared to controls ( $\mathrm{p}=0.04$ and $\mathrm{p}=0.017$, respectively), as demonstrated in table 3 . No statistically significant differences were found between patients and controls in the parieto-occipital white matter and in the prefrontal cortex, regarding either metabolite ratios or absolute concentrations.

In table 4, metabolite ratios of a subgroup (OSA subgroup) of the 22 OSA patients, consisting of those without a history of cardiac disease or cardiovascular risk factors (hypertension, hyperlipidaemia and diabetes mellitus) are compared to controls. This subgroup of 14 OSA patients, without having significant age difference from controls, had statistically significant decreases in NAA/Cr and $\mathrm{Cho} / \mathrm{Cr}$ ratios in frontal white matter when compared to the control group $(\mathrm{p}=0.03$ and 0.01 , respectively). As demonstrated in table 5, Cho concentration was significantly decreased in frontal white matter of the OSA subgroup as compared to controls $(\mathrm{p}=0.03)$, whereas NAA showed only a trend to decrement $(\mathrm{p}=0.09)$. Correlations between metabolite ratios in all three examined areas, and $\mathrm{AHI}, \mathrm{Sa}, \mathrm{O}_{2}$, min, $\mathrm{Sa}, \mathrm{O}_{2}$, mean and $t<90 \%$ were not significant.

In the control group, age showed an inverse correlation only with anterior white matter NAA level $(\mathrm{r}=-0.648$, $\mathrm{p}=0.043$ ). In the same area, the $\mathrm{mI} / \mathrm{Cr}$ ratio of the control group was correlated with age $(r=0.732, p=0.016)$. Metabolite concentration, as well as metabolite ratios, of the patient group did not correlate with age. The effect of interactions of age, ESS, AHI, $S \mathrm{a}, \mathrm{O}_{2}, \mathrm{~min}, S \mathrm{a}, \mathrm{O}_{2}$, mean and $t<90 \%$ on 

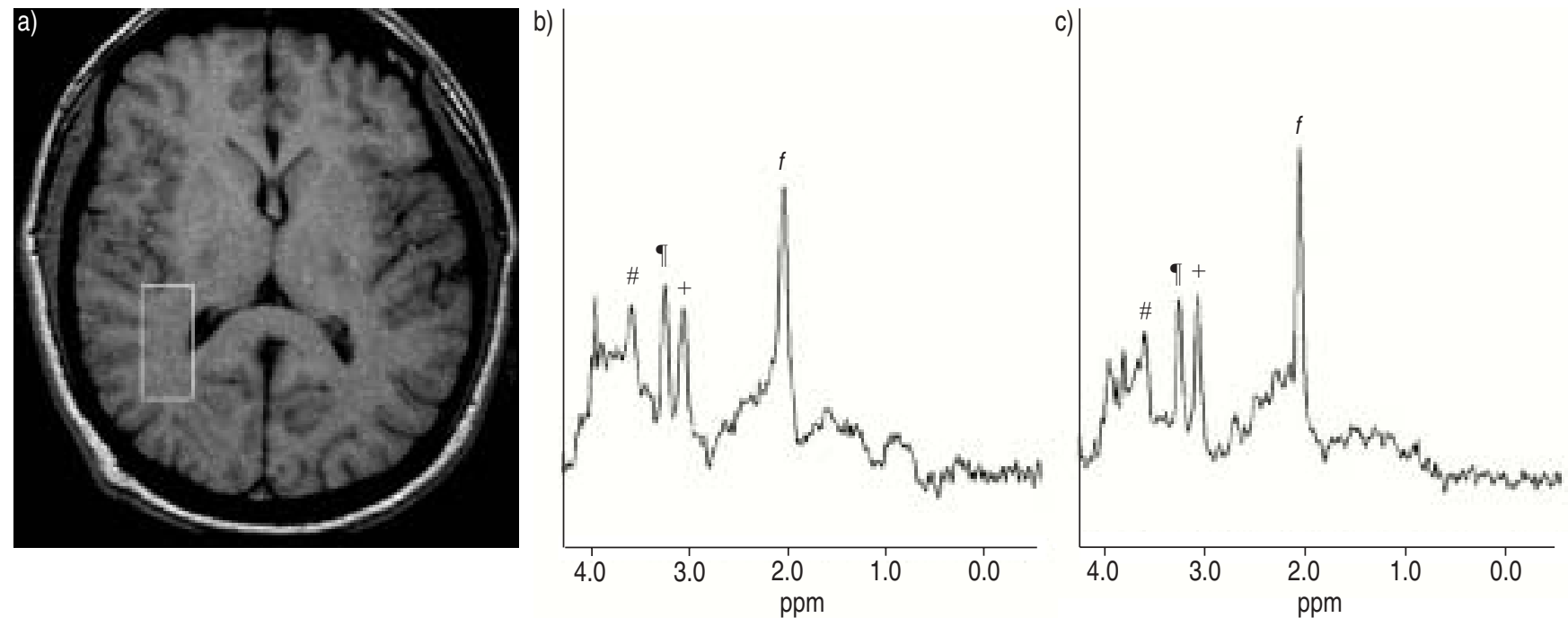

Fig. 1. - a) Location of the examined voxel in the right parieto-occipital white matter (PWM), next to the occipital horn of the lateral ventricle (see box). b) A representative spectra of an obstructive sleep apnoea patient from PWM. c) A representative spectra of a control subject from PWM. ${ }^{\#}$ : myo-inositol; ${ }^{\uparrow}$ : choline; ${ }^{+}$: creatine; ${ }^{f}: N$-acetylaspartate.
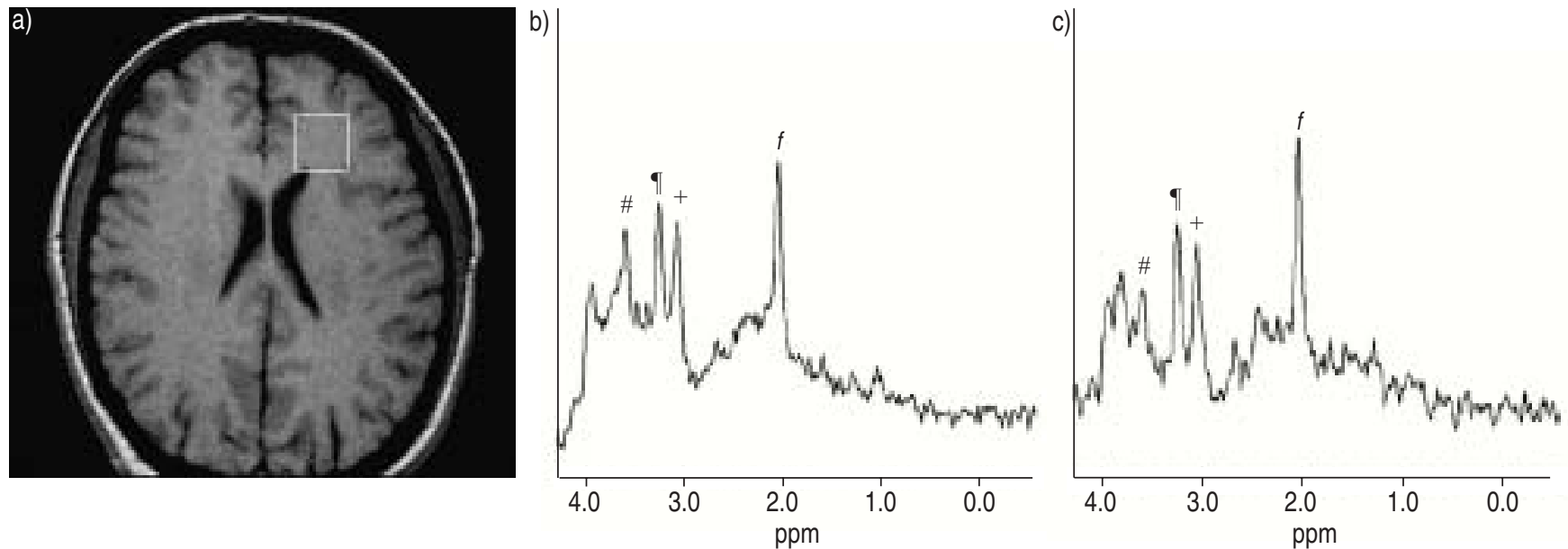

Fig. 2.-a) Location of the examined voxel in the left frontal white matter (FWM), next to the frontal horn of the lateral ventricle (see box) b) A representative spectra of an obstructive sleep apnoea patient from FWM. c) A representative spectra of a control subject from FWM. ${ }^{\#}$ : myo-inositol; ${ }^{\uparrow}$ : choline; ${ }^{+}$: creatine; ${ }^{f}: N$-acetylaspartate.
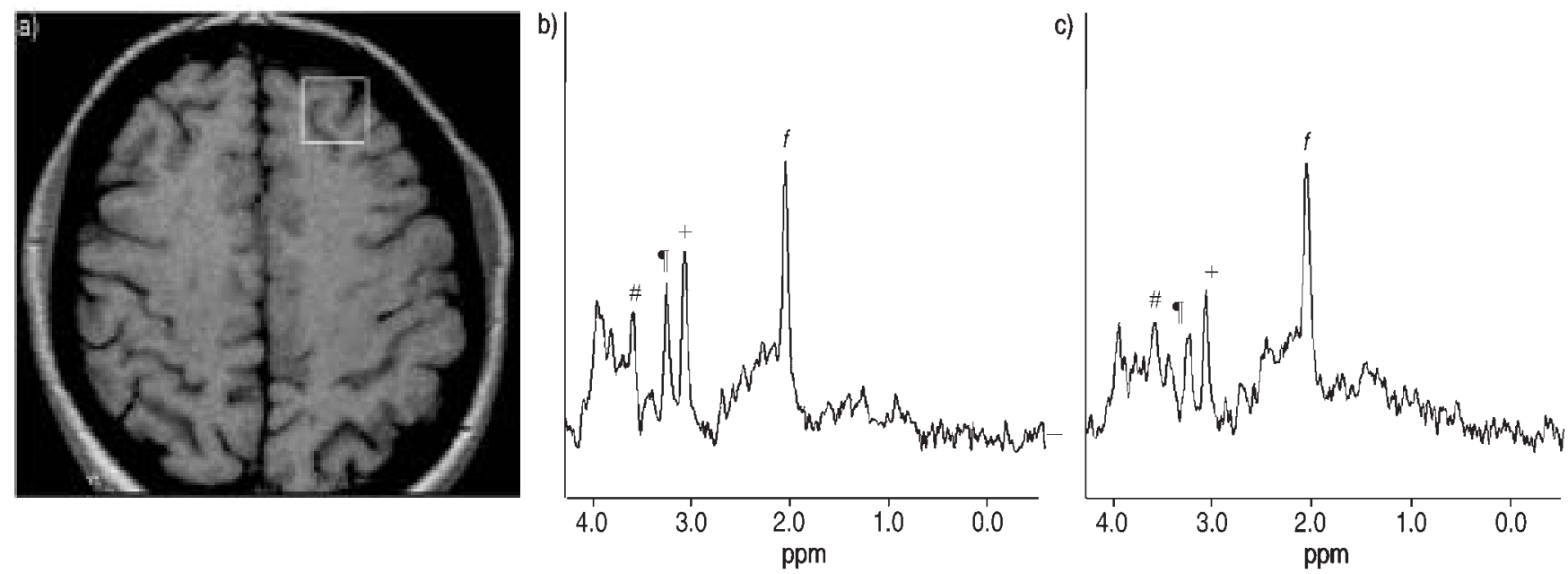

Fig. 3. - a) Location of the examined voxel in the left prefrontal cortex (PFC) (see box). b) A representative spectra of an obstructive sleep apnoea patient from PFC. c) A representative spectra of a control subject from PFC. ${ }^{\#}:$ myo-inositol; ${ }^{\uparrow}$ : choline; ${ }^{+}:$creatine; ${ }^{f}: N$-acetylaspartate. 
Table 1.-Clinical characteristics of obstructive sleep apnoea (OSA) patients and controls

\begin{tabular}{|c|c|c|c|c|c|}
\hline & \multirow[t]{2}{*}{ OSA patients } & \multirow[t]{2}{*}{ OSA subgroup ${ }^{\#}$} & \multirow[t]{2}{*}{ Controls } & \multicolumn{2}{|c|}{ p-value } \\
\hline & & & & OSA to control & Subgroup to controls \\
\hline Subjects $n$ & 22 & 14 & 10 & & \\
\hline Sex & All male & All male & All male & & \\
\hline Age yrs & $49 \pm 9.7$ & $48 \pm 10.1$ & $42.9 \pm 10.5$ & 0.12 & 0.2 \\
\hline AHI events $\cdot h^{-1}$ & $70.6 \pm 19.4$ & $70.1 \pm 19.8$ & $3.4 \pm 1.5$ & $<0.0001$ & $<0.0001$ \\
\hline $\mathrm{Sa}, \mathrm{O}_{2}, \min \%$ & $66.7 \pm 12$ & $65.5 \pm 13.6$ & $94.3 \pm 1.3$ & $<0.0001$ & $<0.0001$ \\
\hline $\mathrm{Sa}_{\mathrm{a}} \mathrm{O}_{2}$, mean $\%$ & $87.7 \pm 5.6$ & $88.1 \pm 6.4$ & $95.7 \pm 0.7$ & $<0.0001$ & $<0.0001$ \\
\hline$t<90 \% \min$ & $122 \pm 84$ & $133.1 \pm 99.5$ & 0 & $<0.0001$ & $<0.0001$ \\
\hline ESS score & $8.2 \pm 3.3$ & $8.4 \pm 2.5$ & & & \\
\hline
\end{tabular}

Data are presented as mean \pm SD. AHI: apnoea hypopnoea index; $S \mathrm{a}, \mathrm{O}_{2}$, min: minimal nocturnal oxyhaemoglobin saturation; $S \mathrm{a}, \mathrm{O}_{2}$, mean: mean nocturnal oxyhaemoglobin saturation; $t<90 \%$ : absolute time of oxyhaemoglobin saturation $<90 \%$ during sleep; ESS: Epworth Sleepiness Scale. ${ }^{\#}$ : OSA patients without history of cardiac disease or cardiovascular risk factors (hypertension, hyperlipidaemia and diabetes mellitus).

Table 2. - Comparison of metabolite ratios between obstructive sleep apnoea (OSA) patients and controls

\begin{tabular}{lccc}
\hline & OSA patients & Controls & p-value \\
\hline Subjects n & 22 & 10 & \\
PWM & & & \\
mI/Cr & $0.68 \pm 0.09$ & $0.69 \pm 0.05$ & 0.92 \\
NAA/Cho & $1.75 \pm 0.32$ & $1.62 \pm 0.28$ & 0.29 \\
NAA/Cr & $1.81 \pm 0.18$ & $1.74 \pm 0.16$ & 0.34 \\
Cho/Cr & $1.07 \pm 0.19$ & $1.09 \pm 0.12$ & 0.44 \\
FWM & & & \\
mI/Cr & $0.66 \pm 0.10$ & $0.73 \pm 0.15$ & 0.14 \\
NAA/Cho & $1.60 \pm 0.29$ & $1.49 \pm 0.25$ & 0.35 \\
NAA/Cr & $1.59 \pm 0.16$ & $1.74 \pm 0.15$ & 0.012 \\
Cho/Cr & $1.01 \pm 0.15$ & $1.19 \pm 0.17$ & 0.008 \\
PFC & & & \\
mI/Cr & $0.64 \pm 0.08$ & $0.62 \pm 0.09$ & 0.49 \\
NAA/Cho & $2.18 \pm 0.35$ & $2.07 \pm 0.36$ & 0.36 \\
NAA/Cr & $1.56 \pm 0.12$ & $1.58 \pm 0.15$ & 0.54 \\
Cho/Cr & $0.74 \pm 0.13$ & $0.78 \pm 0.13$ & 0.45 \\
\hline
\end{tabular}

Data are presented as mean \pm SD. PWM: parieto-occipital white matter; FWM: frontal white matter; PFC: prefrontal cortex; mI: myo-inositol; NAA: $N$-acetylaspartate; Cr: creatine; Cho: choline.

Table 3. - Comparison of absolute metabolite concentrations between obstructive sleep apnoea (OSA) patients and controls

\begin{tabular}{lccl}
\hline & OSA patients & Controls & p-value \\
\hline Subjects n & 22 & 10 & \\
PWM & & & \\
Cho & $1.80 \pm 0.35$ & $1.98 \pm 0.43$ & 0.26 \\
Cr & $4.91 \pm 0.64$ & $5.14 \pm 0.84$ & 0.45 \\
NAA & $7.52 \pm 1.01$ & $7.56 \pm 1.06$ & 0.97 \\
mI & $5.48 \pm 0.74$ & $5.8 \pm 0.93$ & 0.26 \\
FWM & & & \\
Cho & $1.75 \pm 0.33$ & $2.18 \pm 0.44$ & 0.017 \\
Cr & $5.03 \pm 0.69$ & $5.18 \pm 0.64$ & 0.6 \\
NAA & $6.76 \pm 1.06$ & $7.68 \pm 1.06$ & 0.04 \\
mI & $5.53 \pm 1.38$ & $6.16 \pm 1.17$ & 0.18 \\
PFC & & & \\
Cho & $1.41 \pm 0.26$ & $1.57 \pm 0.35$ & 0.25 \\
Cr & $5.55 \pm 0.56$ & $5.66 \pm 0.72$ & 0.57 \\
NAA & $7.32 \pm 0.81$ & $7.6 \pm 0.75$ & 0.36 \\
mI & $5.86 \pm 0.99$ & $5.6 \pm 0.97$ & 0.34 \\
\hline
\end{tabular}

Data are expressed in mmol per $\mathrm{kg}$ and presented as mean \pm SD. PWM: parieto-occipital white matter; FWM: frontal white matter; PFC: prefrontal cortex; Cho: choline; Cr: creatine; NAA: $N$-acetylaspartate; mI: myo-inositol.

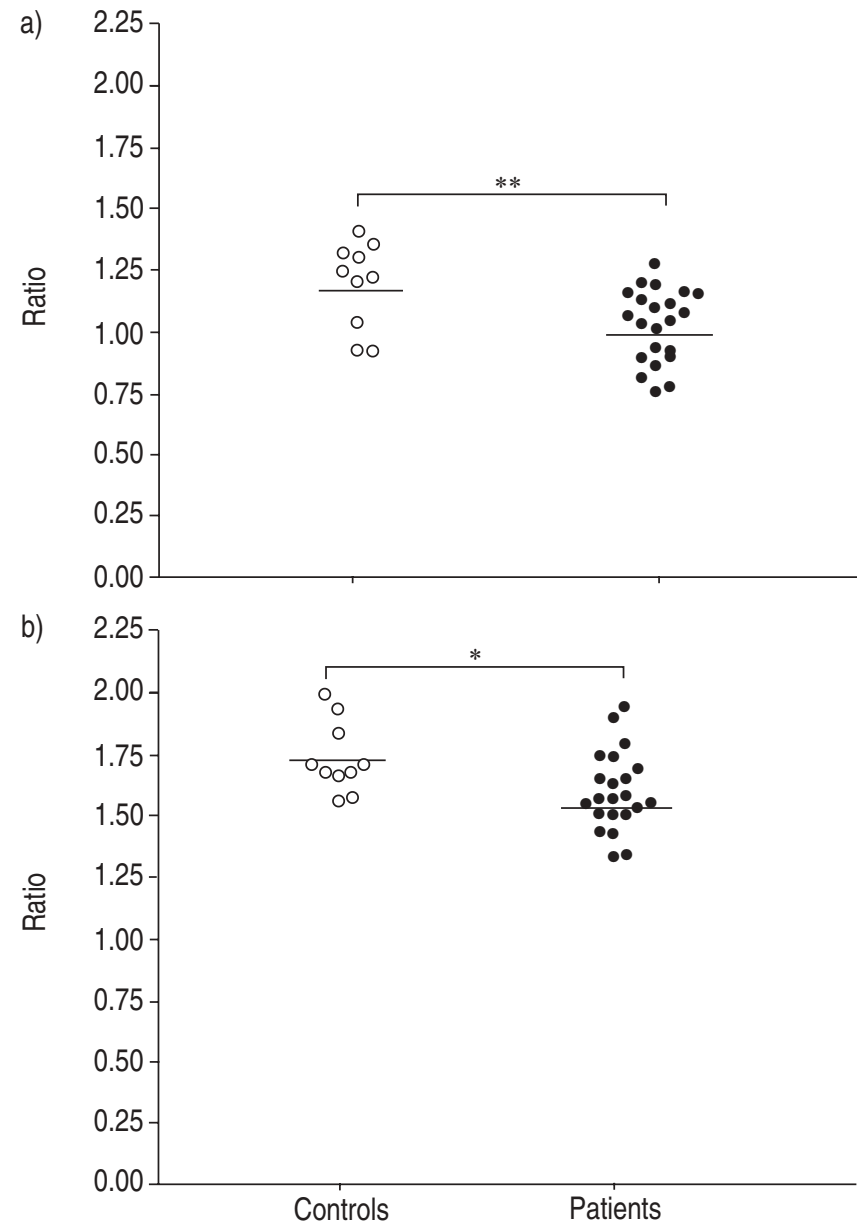

Fig. 4. - Scatterplots of a) choline (Cho)/creatine $(\mathrm{Cr})$ and b) $\mathrm{N}$ acetylaspartate (NAA)/Cr ratios of controls $(\bigcirc)$ and obstructive sleep apnoea (@) patients in frontal white matter. - : mean values. *: $\mathrm{p}<0.05 ; * *: \mathrm{p}<0.01$

metabolic ratios and concentrations was not statistically significant for the control nor for the patient group. ESS was correlated significantly only with NAA/Cho ratio in the posterior white matter $(r=-0.476, p=0.025)$.

\section{Discussion}

In the present study, brain metabolism was investigated in patients with severe OSAS. The results demonstrate a 
Table 4. - Comparison of metabolite ratios between obstructive sleep apnoea (OSA) subgroup (patients without cardiac disease or cardiovascular risk factors) and controls

\begin{tabular}{lccc}
\hline & OSA subgroup & Controls & p-value \\
\hline $\begin{array}{l}\text { Subjects n } \\
\text { PWM }\end{array}$ & 14 & 10 & \\
mI/Cr & $0.7 \pm 0.08$ & $0.69 \pm 0.05$ & 0.56 \\
NAA/Cho & $1.75 \pm 0.30$ & $1.62 \pm 0.28$ & 0.36 \\
NAA/Cr & $1.84 \pm 0.19$ & $1.74 \pm 0.16$ & 0.27 \\
Cho/Cr & $1.07 \pm 0.2$ & $1.09 \pm 0.12$ & 0.77 \\
FWM & & & \\
mI/Cr & $0.65 \pm 0.1$ & $0.73 \pm 0.15$ & 0.15 \\
NAA/Cho & $1.66 \pm 0.31$ & $1.49 \pm 0.25$ & 0.22 \\
NAA/Cr & $1.61 \pm 0.13$ & $1.74 \pm 0.15$ & 0.03 \\
Cho/Cr & $1.0 \pm 0.17$ & $1.19 \pm 0.17$ & 0.01 \\
PFC & & & \\
mI/Cr & $0.63 \pm 0.08$ & $0.62 \pm 0.09$ & 0.49 \\
NAA/Cho & $2.19 \pm 0.40$ & $2.07 \pm 0.36$ & 0.44 \\
NAA/Cr & $1.56 \pm 0.11$ & $1.58 \pm 0.15$ & 0.64 \\
Cho/Cr & $0.74 \pm 0.13$ & $0.78 \pm 0.13$ & 0.5 \\
\hline
\end{tabular}

Data are presented as mean \pm SD. PWM: parieto-occipital white matter; FWM: frontal white matter; PFC: prefrontal cortex; mI: myo-inositol; NAA: $N$-acetylaspartate; $C r$ : creatine; Cho: choline.

Table 5. - Comparison of metabolite concentrations between obstructive sleep apnoea (OSA) subgroup (patients without cardiac disease or cardiovascular risk factors) and controls

\begin{tabular}{lccc}
\hline & OSA subgroup & Controls & p-value \\
\hline Subjects n & 14 & 10 & \\
PWM & & & \\
Cho & $1.80 \pm 0.37$ & $1.97 \pm 0.42$ & 0.27 \\
Cr & $4.89 \pm 0.65$ & $5.13 \pm 0.84$ & 0.36 \\
NAA & $7.50 \pm 0.79$ & $7.56 \pm 1.05$ & 0.97 \\
mI & $5.55 \pm 0.70$ & $5.82 \pm 0.88$ & 0.46 \\
FWM & & & \\
Cho & $1.74 \pm 0.36$ & $2.17 \pm 0.44$ & 0.03 \\
Cr & $5.11 \pm 0.77$ & $5.18 \pm 0.63$ & 0.97 \\
NAA & $6.90 \pm 1.13$ & $7.67 \pm 1.06$ & 0.09 \\
mI & $5.57 \pm 1.5$ & $6.16 \pm 1.17$ & 0.27 \\
PFC & & & \\
Cho & $1.39 \pm 0.18$ & $1.56 \pm 0.35$ & 0.18 \\
Cr & $5.49 \pm 0.6$ & $5.65 \pm 0.71$ & 0.50 \\
NAA & $7.19 \pm 0.64$ & $7.60 \pm 0.75$ & 0.20 \\
mI & $5.74 \pm 0.93$ & $5.59 \pm 0.96$ & 0.46 \\
\hline
\end{tabular}

Data are expressed in mmol per $\mathrm{kg}$ and presented as mean \pm SD. PWM: parieto-occipital white matter; FWM: frontal white matter; PFC: prefrontal cortex; Cho: choline; Cr: creatine; NAA: $N$-acetylaspartate; mI: myo-inositol.

significant decrease in $\mathrm{NAA} / \mathrm{Cr}$ and $\mathrm{Cho} / \mathrm{Cr}$ ratios, as well as a reduction in absolute concentrations of NAA and Cho, in the FWM of OSA patients when compared to controls.

NAA is an intraneuronal molecule and, in the mature brain, it is found only in neurons and axons. NAA is reduced in many brain disorders, in the presence of neuronal and/or axonal loss or dysfunction, such as infarcts, dementia, brain tumours, hypoxic encephalopathy and multiple sclerosis [7, 8].

The $\mathrm{Cr}$ signal is generated by the sum of creatine and phosphocreatine, and reflects energy metabolites. Because this peak remains relatively stable, it is frequently used as a reference peak to normalise metabolite signal intensities.

$\mathrm{mI}$ originates almost exclusively from glial cells. Elevated $\mathrm{mI}$ represents both the accumulation of myelin breakdown products and astrocytosis [7, 8, 13].

The Cho peak measures total levels of mobile choline, which include free choline, glycerophosphocholine (a byproduct of phosphatidylcholine breakdown), phosphocholine (a phosphatidylcholine precursor) and minute amounts of acetylcholine. Elevated Cho represents increased membrane turnover or increased cellular density, and it has been reported in cases of active demyelination, brain tumours and glial proliferation [7, 8, 13].

Decreased NAA in the frontal white matter of OSA patients indicates axonal loss and/or dysfunction [7, 8]. The deep white matter, where metabolic impairment was found in the current study, seems to be selectively affected in OSAS. Previous studies applying MRS with chemical shift imaging have demonstrated a decrease in the NAA/Cho ratio in the posterior periventricular white matter $[9,10]$ and lactate production in the centrum semiovale of OSA patients during sleep, indicating that hypoxia is causing anaerobic glycolysis [14]. Absolute concentrations were not calculated in these studies, so it is not clear if the decreased ratio was due to a decrease in NAA and/or an elevation in Cho. A recent study, using computed tomography, demonstrated that white matter disease severity in patients with acute stroke and OSAS correlated independently with AHI [15]. One possible explanation is that the arterial supply of the deep white matter is not sufficient to compensate for the decreased and fluctuating cerebral perfusion and the impaired cerebral vascular autoregulation that have been reported in OSA patients $[10,16$, 17]. In fact, the arterial networks of the deep white matter, the so-called internal border zone, are terminals without collaterals or anastomoses [18].

An interesting finding in this study is the decrement of Cho in the frontal white matter of OSA patients. Decreased Cho has been reported in mitochondrial, hypomyelinotic and metabolic diseases, hepatic encephalopathy [7, 8], Grave's disease [19], Lewy body dementia [20], and chronic obstructive pulmonary disease [21]. It has been suggested that decreased in vivo Cho levels indicate loss of myelin lipids or phospholipid metabolism dysfunction [17, 18, 22]. These results are in agreement with the report of SHIM et al. [21]. These investigators found a reduction in NAA, $\mathrm{Cr}$ and Cho concentrations in the parietal white matter of COPD patients with resting normoxaemia and nocturnal desaturation, a respiratory profile similar to OSAS. A previous study has demonstrated a decrease in Cho metabolites as a consequence of brief ischaemic episodes in an experimental animal model [22]. Nevertheless, if chronic haemodynamic impairment were the sole pathogenic factor related to OSA, one would expect elevation of Cho and perhaps of $\mathrm{mI}$ due to gliosis and myelin breakdown [13, 18, 23]. It is suggested here that a possible interpretation for the Cho decrement is that OSA does not promote gliosis but induces brain metabolic impairment through a unique combination of fluctuating haemodynamic impairment, sleep fragmentation and intermittent hypoxia. Experimental intermittent hypoxia per se can induce neuronal apoptosis and biochemical changes in animals' brains, perhaps in a different manner than chronic sustained hypoxia $[24,25]$. In addition, recent studies have shown that the prefrontal areas are especially sensitive to sleep deprivation and recovery sleep, presumably because these regions have a unique requirement for sleep-related recovery $[6,26]$ and that sleep deprivation can alter Cho metabolism. DORSEY et al. [27] found an increase of glycerophosphocholine concentration in healthy volunteers after the recovery night, following a night of sleep deprivation [27]. In the current study, although the patients did not have severe sleepiness, ESS score is correlated with NAA/Cho ratio, but only in the posterior white matter.

OSA patients frequently have a combination of vascular risk factors, including hypertension, diabetes mellitus, hyperlipidaemia and central obesity [28]. All these factors are 
associated with increased risk of stroke and could possibly promote brain metabolic impairment. Nevertheless, in the present study, the subgroup of OSA patients without a medical history of cardiac disease or cardiovascular risk factors also had significantly depressed NAA/Cr and $\mathrm{Cho} / \mathrm{Cr}$ ratios, as well as a diminished Cho concentration in frontal white matter, when compared to controls. This finding suggests that OSAS may promote brain metabolic impairment even in the absence of cardiovascular co-morbidities.

Frontal periventricular white matter lesions, to the best of the current authors' knowledge, have not been reported previously in OSA patients without a history of stroke. Frontal white matter lesions could be associated with the neuropsychological deficits that complicate OSAS. Patients with OSAS frequently have mild impairment in attentionconcentration, memory and, in particular, executive functions, such as problem solving, planning of goal-oriented behaviour and mental flexibility, which are sometimes irreversible, even after nCPAP treatment $[1-4,6]$. Cognitive executive functions are associated with the prefrontal cortex and prefrontal-subcortical brain circuits [5], thus it has been proposed that OSAS induces chemical and structural cellular injury, affecting the prefrontal cortex $[4,6]$. In the study presented here, no metabolic impairment was found in the examined prefrontal cortex, but it was demonstrated that patients with severe OSAS have metabolic impairment in the frontal white matter. It is, therefore, suggested that these findings may offer an explanation for the specific pattern of cognitive deficits frequently reported in OSA patients, as it is well known that anterior white matter lesions can induce executive dysfunction by interrupting prefrontal-subcortical circuits $[5,29,30]$.

An important limitation to this study is the lack of neuropsychological measures in the examined patients, although is well documented that frontal lobe-mediated executive functions are usually impaired in patients with severe OSAS [1]. A second limitation is that, because only patients with severe OSA were included, there were not enough variances in the respiratory parameters that could predict the metabolic changes. KAMBA et al. [10], who studied OSA patients with wide range of severity (mean AHI $43.78 \pm 30$ ), found a significant negative association between AHI and NAA/Cho ratio for the posterior cerebral white matter. A third consideration is about the effect of aging on brain metabolism. MRS studies on the effect of aging on brain metabolites are often discrepant. Results of quantitative MRS studies vary from increased $\mathrm{Cr}$ and Cho levels to unchanged or decreased NAA levels and lack of any significant change $[22,31,32]$. In the present study, anterior white matter NAA levels of the OSA patient group did not show a significant correlation with aging, but the anterior white matter NAA level of the control group was inversely correlated with aging. These data suggest that, although the age difference between the patient and the control group was not statistically significant, it could be responsible for some of the reported NAA decrement. Nevertheless, if the results were biased by some kind of age effect, one would expect, according to the literature, a decrement in NAA and an increment in Cho, or vice versa, and not a decrement in both metabolites.

In conclusion, the results of this study demonstrate that severe obstructive sleep apnoea syndrome can promote axonal loss or dysfunction, as well as myelin metabolism impairment in the frontal periventricullar white matter. These lesions are in the territory of crucial frontal-subcortical circuits and they could be associated with the, sometimes irreversible, cognitive executive deficits reported in obstructive sleep apnoea patients. Further studies are needed to confirm whether there is a direct correlation between frontal metabolic dysfunction and cognitive impairment in obstructive sleep apnoea patients, and to examine the reversibility of the spectroscopic abnormalities after nasal continuous positive airway pressure treatment.

\section{References}

1. Decary A, Rouleau I, Monplaisir J. Cognitive deficits associated with sleep apnoea syndrome: a proposed neuropsychological test battery. Sleep 2000; 23: 369-381.

2. Engelman HM, Kingshot RN, Martin SE, Douglas NJ. Cognitive function in the sleep apnoea/hypopnoea syndrome (SAHS). Sleep 2000; 23: s102-s108.

3. Naegele B, Pepin JL, Levy P, Bonnet C, Pellat J, Feuerstein C. Cognitive executive dysfunction in patients with obstructive sleep apnoea syndrome (OSAS) after CPAP treatment. Sleep 1998; 21: 392-397.

4. Bedard MA, Monplaisir J, Malo J, Richer F, Rouleau I. Persistent neuropsychological deficits and vigilance impairment in sleep apnoea syndrome after treatment with continuous positive airway pressure (CPAP). J Clin Exp Neuropsychol 1993; 15: 330-341.

5. Cummings JL. Frontal-subcortical circuits and human behaviour. Arch Neurol 1993; 50: 873-880.

6. Beebe DW, Gozal D. Obstructive sleep apnoea and the prefrontal cortex: towards a comprehensive model linking nocturnal upper airway obstruction to daytime cognitive and behavioural deficits. $J$ Sleep Res 2002; 11: 1-16.

7. Castillo M, Kwock L, Mukherji SK. Clinical applications of proton MR spectroscopy. AJNR Am J Neuroradiol 1996; 17 : $1-15$.

8. Rudkin TM, Arnold DL. Proton magnetic resonance spectroscopy for the diagnosis and management of cerebral disorders. Arch Neurol 1999; 56: 919-926.

9. Kamba M, Suto Y, Ohta Y, Inoue Y, Matsuda E. Cerebral metabolism in sleep apnoea: evaluation by magnetic resonance spectroscopy. Am J Resp Crit Care Med 1997; 156: 296-298.

10. Kamba M, Inoue Y, Higami S, Suto Y, Ogawa T, Chen W. Cerebral metabolic impairment in patients with obstructive sleep apnoea syndrome: an independent association of obstructive sleep apnoea with white matter change. $J$ Neurol Neurosurg Psychiatry 2001; 71: 334-339.

11. American Thoracic Society. Indications and standards for cardiopulmonary sleep studies. Am Rev Respir Dis 1989; 139: 559-568.

12. Webb PG, Sailasuta N, Kohler SJ, Raidy T, Moats RA, Hurd RE. Automated single-voxel proton MRS: technical development and multisite verification. Magn Reson Med 1994; 31: 365-373.

13. Bitsch A, Bruhn H, Vougioukas V, et al. Inflammatory CNS demyelination: histopathologic correlation with in vivo quantitative proton MR spectroscopy. AJNR Am J Neuroradiol 1999; 20: 1619-1627.

14. Kamba M, Inoue Y, Higami S, Suto Y. Age-related changes in cerebral lactate metabolism in sleep-disordered breathing. Neurobiol Aging 2003; 24: 753-760.

15. Harbison J, Gibson GJ, Birchall D, Zammit-Maempel I, Ford GA. White matter disease and sleep-disordered breathing after acute stroke. Neurology 2003; 61: 959-963.

16. Leung RST, Bradley TD. Sleep apnoea and cardiovascular disease. Am J Respir Cri Care Med 2001; 164: 2147-2165.

17. Derdeyn CP, Khosla A, Videen TO, et al. Severe hemodynamic impairment and border zone-region infraction. Radiology 2001; 220: 195-201.

18. Pantoni L, Garcia JH. Pathogenesis of leukoaraiosis: a review. Stroke 1997; 28: 652-659.

19. Elberling TV, Danielsen ER, Rasmussen AK, FeldtRasmussen U, Waldemar G, Thomsen C. Reduced myoinositol and total choline measured with cerebral MRS in 
acute thyrotoxic Graves' disease. Neutology 2003; 60: 142145.

20. Molina JA, Garcia-Segura JM, Benito-Leon J, et al. Proton magnetic resonance spectroscopy in dementia with Lewy bodies. Eur Neurol 2002; 48: 158-163.

21. Shim TS, Lee JH, Kim SY, et al. Cerebral metabolic abnormalities in COPD patients detected by localized proton magnetic resonance spectroscopy. Chest 2001; 120: 15061513.

22. Sijens PE, den Heijer T, Origgi D, et al. Brain changes with aging: MR spectroscopy at supraventricular plane shows differences between women and men. Radiology 2003; 226: 889-896.

23. Brooks WM, Mary MH, Kodituwakku PW, Garry PJ, Rosenberg GA. ${ }^{1}$ H-MRS differentiates white matter hyperintensities in subcortical arteriosclerotic encephalopathy from those in normal elderly. Stroke 1997; 28: 19401943.

24. Reeves SR, Gozal E, Guo SZ, et al. Effect of long-term intermittent and sustained hypoxia on hypoxic ventilatory and metabolic responses in the adult rat. J Appl Physiol 2003; 95: 1767-1774.

25. Douglas RM, Xue J, Chen JY, Haddad CG, Alper SL, Haddad GG. Chronic intermittent hypoxia decreases the expression of $\mathrm{Na} / \mathrm{H}$ exchangers and $\mathrm{HCO} 3$ - dependent transporters in mouse CNS. J Appl Physiol 2003; 95: 292-299.
26. Thomas M, Sing H, Belenky G, et al. Neural basis of alertness and cognitive performance impairments during sleepiness. I. Effects of $24 \mathrm{~h}$ of sleep deprivation on waking human regional brain activity. J Sleep Res 1999; 9: 335-352.

27. Dorsey CM, Lukas SE, Moore CM, Parow AM, Villafuerte RA, Renshaw PF. Phosphorous 31 magnetic resonance spectroscopy after total sleep deprivation in healthy adult men. Sleep 2003; 26: 573-577.

28. Wilkox I, McNamara SG, Collins FI, Grunstein RR, Sullivan CE. "Syndrome Z": the interaction of sleep apnoea, vascular risk factors and heart disease. Thorax 1998; 53: S25-S28

29. Royall DR. Executive cognitive impairment: a novel prospective on dementia. Neuroepidemiology 2000; 19: 293299.

30. Kramer JH, Reed BR, Mungas D, Weiner MW, Chui HC. Executive dysfunction in subcortical ischaemic vascular disease. J Neurol Neurosurg Psychiatry 2002; 72: 217-220.

31. Leary $\mathrm{S}$, Brex $\mathrm{P}$, MacManus $\mathrm{D}$, et al. A ${ }^{1} \mathrm{H}$ magnetic resonance spectroscopy study of aging in parietal white matter; implications for trials in multiple sclerosis. Magn Reson Imaging 2000; 18: 455-459.

32. Brooks JCW, Roberts N, Kemp GJ, Gosney MA, Lye M, Whithouse GH. A proton magnetic resonance spectroscopy study of age-related changes in frontal lobe metabolite concentrations. Cerebral Cortex 2001; 11: 598-605. 